\title{
Towards Identification of Individual Etiologies by Resolving Genomic and Biological Conundrums in Patients with Autism Spectrum Disorders
}

\author{
M. Poot \\ Department of Medical Genetics, University Medical Center Utrecht, Utrecht, The Netherlands
}

\section{Key Words}

Autism - Copy number variation - Personalized medicine . Polygenic mode . Single nucleotide variation - Transgenic mouse models

\begin{abstract}
Recent genomic research into autism spectrum disorders (ASD) has revealed a remarkably complex genetic architecture. Large numbers of common variants, copy number variations and single nucleotide variants have been identified, yet each of them individually afforded only a small phenotypic impact. A polygenic model in which multiple genes interact either in an additive or a synergistic way appears the most plausible for the majority of ASD patients. Based on recently identified ASD candidate genes, transgenic mouse models for neuroligins/neurorexins and genes such as Cntnap2, Cntn5, Tsc1, Tsc2, Akt3, Cyfip1, Scn1a, En2, Slc6a4, and $B c k d k$ have been generated and studied with respect to behavioral and neuroanatomical phenotypes and sensitivity to drug treatments. From these models, a few clues for potential pharmacologic intervention emerged. The Fmr1, Shank2 and Cntn5 knockout mice exhibited alterations of glutamate receptors, which may become a target for pharmacologic modulation. Some of the phenotypes of Mecp2 knockout mice can be ameliorated by administering IGF1. In
\end{abstract}

the near future, comprehensive genotyping of individual patients and siblings combined with the novel insights generated from the transgenic animal studies may provide us with personalized treatment options. Eventually, autism may indeed turn out to be a phenotypically heterogeneous group of disorders ('autisms') caused by combinations of changes in multiple possible candidate genes, being different in each patient and requiring for each combination of mutations a distinct, individually tailored treatment.

Copyright $\odot 2013$ S. Karger AG, Basel

Within the realm of neurodevelopmental diseases, autism spectrum disorders (ASD) are typified by concomitant deficits in 3 domains of behavior: reciprocal social interaction, communication, and stereotyped and restricted behaviors. When deficits are present in all 3 domains with at least one defect in one area being detected before completion of 3 years of age, the patient is classified as autistic disorder [American Psychiatric Association, 2000]. This is the narrower diagnostic category within the spectrum of ASD, which also includes Asperger syndrome and Pervasive Developmental DisorderNot Otherwise Specified (PDD-NOS). ASDs occur either sporadically or as familial cases with an estimated prevalence of 1/150 children [Fombonne, 2009]. Males are 4

\section{KARGER}

E-Mail karger@karger.com

www.karger.com/msy
(C) 2013 S. Karger AG, Basel

$1661-8769 / 13 / 0045-0213 \$ 38.00 / 0$
Dr. Martin Poot

Department of Medical Genetics, University Medical Center Utrecht Str. 1.505

NL-3508 AB Utrecht (The Netherlands)

E-Mail m.poot@umcutrecht.nl 
times more frequently affected than females [Newschaffer et al., 2007]. In 15-70\% of children with ASD, also mental and developmental retardation has been diagnosed [Toro et al., 2010]. Comparisons of monozygotic and dizygotic twins suggested a heritability as high as $90 \%$ for the narrow phenotype of autistic disorder [Bailey et al., 1995; Le Couteur et al., 1996; Freitag, 2007; Skuse, 2007; Hallmayer et al., 2011].

ASD may occur in a sporadic patient in an otherwise healthy family, i.e. a simplex family, or in several children of one generation in the so-called multiplex families. Analyzing autism risk in multiplex families from the Autism Genetic Resource Exchange, Zhao et al. [2007] found strong evidence for dominant transmission to male offspring. The authors hypothesized that actually 2 types of families may exist: low-risk families with sporadic autism patients being mainly caused by de novo mutations with high penetrance in males and relatively poor penetrance in females, and high-risk families in which ASD probands received a dominant mutation, most often from females, who carry this mutation but are themselves unaffected [Zhao et al., 2007]. Using a Bayesian approach in a population-based sample, Nishiyama et al. [2009] showed that the largest proportion of ASD cases can be explained by a model allowing multiple, concomitantly inherited risk alleles. This suggested a polygenic model of ASD causation, which is appealing in view of the complex clinical phenotype of ASD.

\section{Genetic Architecture of Autism}

ASD may be part of Mendelian syndromes, such as the Rett and the Smith-Lemli-Opitz syndrome, resulting from genomic rearrangements [Vorstman et al., 2006], or from mutations in a putative 'ASD risk gene', e.g. DISC1, NRXN1, RELN, CNTNAP2, and MECP2 [Abrahams and Geschwind, 2008]. The rapid development of novel methods for genetic analysis has produced large amounts of data, which necessitate rethinking our assumptions regarding the genetic architecture of ASD and other neurobehavioral disorders [State and Levitt, 2011]. Thus, classical models, such as the common disease-common variant hypothesis, have been supplemented by views implicating rare variants with stronger phenotypic impact and by polygenic models that incorporate combinations of variants with mixed effect strength.

Analysis of rare, often de novo structural chromosome variation of ASD patients indicated numerous regions and loci for ASD candidate genes with a strong pheno- typic impact [Vorstman et al., 2006]. This prompted investigators to perform genome wide segmental aneuploidy profiling, which revealed numerous submicroscopic structural genome alterations, named copy number variations (CNVs). Such CNVs were either inherited or emerged de novo in 7-27\% of ASD patients under investigation [Jacquemont et al., 2006; Autism Genome Project Consortium et al., 2007; Sebat et al., 2007; Marshall et al., 2008; Cuscó et al., 2009]. In a recent study of the Autism Genetic Resource Exchange dataset, Itsara et al. [2009] found a de novo CNV rate in the order of $2 \%$ and concluded that de novo CNVs may contribute significantly to autism risk. Patients with ASD in addition to comorbidities (e.g. dysmorphisms or congenital anomalies) and a family history of intellectual impairment not only made up the majority of cases, they are also more likely to carry CNVs [Marshall et al., 2008; Toro et al., 2010]. On the basis of these data, the number of ASDrelated $\mathrm{CNV}$ regions in the human genome has been estimated at 130-234 [Sanders et al., 2011]. In multiplex ASD families, potentially pathogenic CNVs often did not cosegregate with disease [Bucan et al., 2009; Glessner et al., 2009; Holt et al., 2010; Salyakina et al., 2011; van Daalen et al., 2011]. This further underscores the complex genetic architecture of ASD and suggests that additional alleles may be involved.

Searching for specific ASD risk genes, genetic linkage and association studies identified only few of such genes and contributed little to explain the phenotypic variability among ASD patients. Moreover, replication of results proved to be difficult [Vorstman et al., 2006; Autism Genome Project Consortium et al., 2007; Freitag, 2007; Abrahams and Geschwind, 2008; Toro et al., 2010], yet common variants with odds ratios around 1.3 in genes such as CDH9, CDH10 and NRXN have been identified [Autism Genome Project Consortium et al., 2007; Ma et al., 2009; Wang et al., 2009]. Overall, individual common variants exert weak effects on the risk for ASD, such that all these variants together may explain only a modest part of the genetic component of the disease [Anney et al., 2012]. Reviewing the genetic architecture of ASD, Devlin and Scherer [2012] estimated that ASD-related syndromes accounted for roughly $10 \%$ of all cases, while rare chromosomal abnormalities, CNVs and rare, penetrant mutations in genes may contribute to another $15 \%$ of cases. This would leave us with approximately $75 \%$ of ASD cases without known genetic etiology. In other words, there is an appreciably amount of missed heritability in ASD and further variants are left to be found [Manolio et al., 2009]. 
Recent whole exome sequencing studies of over 1,000 ASD families revealed a de novo rate of potentially relevant single nucleotide variants (SNVs) in the order of $1-2 \cdot 10^{8}$ per nucleotide and per patient [Iossifov et al., 2012; Neale et al., 2012; O’Roak et al., 2012; Sanders et al., 2012]. Although these 4 exome sequencing studies discovered hundreds of de novo SNVs, only 3 genes, SCN2A [Sanders et al., 2012], NTNG1 and CHD8 [O'Roak et al., 2012], carried a SNV in more than a single patient. Together, these exome sequencing studies point toward hundreds of potential ASD risk genes.

Based on studies of CNVs, a model of ASD etiology, assuming a single or several loci with additive effects, has been proposed [Cook and Scherer, 2008]. In this model, a CNV by itself may be sufficient to cause ASD or may contribute additional effect(s) to other mutation(s) such that via an additive interaction the threshold for fullblown ASD will be crossed [Cook and Scherer, 2009]. Alternatively, Gillberg and Coleman [1996] proposed that autism may be 'a syndrome or series of syndromes caused by many different separate individual diseases'. This proposal easily incorporates the finding that the majority of patients with ASD suffer from intellectual abilities [Toro et al., 2010] and show problems with processing of visual and auditory stimuli [Vlamings et al., 2010; Magnée et al., 2011]. If this model was to be true, we would have to search for simultaneously occurring mutations in several genes related to each of several partial or endophenotypes of the patient. The study by Pinto et al. [2010] of CNVs in ASD and a case with CNTNAP2 disruption and additional CNVs [Poot et al., 2010a] are both consistent with this model. In a large cohort of 854 ASD families, Leblond et al. [2012] found 3 patients with a de novo loss of SHANK2, which encodes a scaffolding protein located in the postsynaptic density part of glutaminergic synapses. Interestingly, 2 cases showed an additional gain of the nicotinic receptor CHRNA7 and 1 case a loss of CYFIP1. This study supports a model of epistatic interactions of genes in distinct biological pathways, which upon disruption may lead to ASD. These bits of evidence indicate that studies aimed at comprehensive testing for possible disruption of epistatic networks as the underlying mechanism for ASD will become necessary.

\section{Implications for Medical Genetics}

In clinical practice, medical genetics is concerned with 2 basic questions: first, what is the cause (etiology) of the disorder and second, if genetic, what is its recurrence risk? If the cause can be unambiguously identified, possible treatment(s) can be envisaged. The recurrence risk may influence the reproductive behavior of the parents and other members of their kinship. Therefore, an estimate of the recurrence risk constitutes actionable genetic information.

The overview of the genetic architecture of ASD, given above, indicates that identification of 'the' causal mutation may be an inappropriate and even futile effort. Once a CNV or a SNV has been identified, one cannot be certain that other, undetected variants may coexist and contribute to the disorder of the patient [Girirajan et al., 2012]. Halting all genetic investigations after detection of the first variant has been loosely termed 'diagnostic fatalism' [Poot et al., 2011]. Such diagnostic fatalism would preclude one from searching further. Indeed, in cases in which 'the' (single) mutation has been reported, this may represent such an ascertainment bias, i.e. missed heritability, rather than a case of a single, fully penetrant, causative mutation.

Yet, considerations regarding possibly missed heritability should not stop us from interpreting the phenotypic, pedigree and mutation data we actually have. To answer the question for 'the cause' of the ASD phenotype of the patient, we first have to determine whether this is likely to be a sporadic patient or may be one of several affected children in a multiplex family. In a sporadic case, a de novo CNV or SNV may very well by itself be pathogenic. Yet, there are 2 caveats to be considered: first, if the affected child is the first born or the only ASD patient in the family, 'reproductive stoppage' may have prevented formation of a multiplex family [Zhao et al., 2007]. If the patient appeared in a truly simplex family, the child is sporadic and a de novo CNV or SNV may be sufficient cause. If, however, reproductive stoppage has occurred, we need to look further. The latter constellation may exist if, first, there is evidence for communicative and social impairments in the family as ascertained by applying the Social Responsiveness Scale (SRS) to the entire family [van Daalen et al., 2011]. If one or both parents score 1 or even 2 SD above the normal range on the SRS, i.e. show evidence for subclinical perturbation of social interaction, this suggests that multiple pathogenic alleles may exist within this particular family [van Daalen et al., 2011]. A second hint may be the age of the father at conception [Buizer-Voskamp et al., 2011]. In this population registry-based study, fathers $>40$ years of age showed a 3.3 times increased odd of having a child with ASD as compared to fathers $<20$ years of age. The authors attributed this elevated age at conception to a putative delay in so- 
cial integration and life cycle development of males with subclinical mental impairments [Buizer-Voskamp et al., 2011]. With the age of conception, the risk of de novo SNVs increases [Kong et al., 2012]. This adds insult to injury, i.e. the reproductive delay due to mental problems of the father gets compounded by a de novo SNV in the offspring. In such situations, a polygenic model, consisting of a combination of transmitted alleles and a de novo SNV, may apply. Reproductive stoppage after the first child with ASD on the other hand will mask a constellation with potentially transmitted dominant risk alleles as pointed out by Zhao et al. [2007] and, hence, an appreciable, yet underestimated, recurrence risk.

In their study, they focused on large ASD families in which reproductive stoppage has been excluded. In these particular families, which made up the majority of their sample, evidence for dominant transmission form a healthy mother onto affected sons was found. Their selection procedure was necessary to avoid confounding factors, yet inevitably harboring a selection bias. The population-based study of Nishiyama et al. [2009] indicated that in the majority of ASD families, multiple genetic etiologies may occur. These findings suggest that the model of Zhao et al. [2007] may actually be widespread among families with ASD. This adds impetus to studies, which found complex inheritance patterns in the families studied [Marshall et al., 2008; Bucan et al., 2009; Salyakina et al., 2011; van Daalen et al., 2011]. In a significant number of families, these studies found evidence for contributing alleles, which were, by themselves, not sufficient to fully explain the ASD in the patient(s). Yet, many of the CNVinvolved genes either had been associated with ASD or with intellectual delay [Pinto et al., 2010; Poot et al., $2010 \mathrm{~b}]$. On the one hand, this makes it increasingly more difficult to attribute CNVs and SNVs specifically to ASD. On the other hand, this corroborates a strong overlap between ASD and intellectual delay, and possibly other neurobehavioral disorders, as has been indicated by several authors [Girirajan and Eichler, 2010; Toro et al., 2010; Poot et al., 2011].

\section{Validation of Putatively Contributing Candidate Genes}

Contributing CNVs and SNVs may provide valuable clues toward subtypes of ASD and subsequently toward individualized treatment options. The genes affected by such CNVs and SNVs may be part of specific biological networks or biochemical pathways [Pinto et al., 2010]. If mutated, the role these genes play in their respective biological network is changed and the functioning of the network as a whole may become altered. Such altered functioning may by itself not be sufficient to elicit ASD, but may entail (neurological) impairments that eventually could contribute to ASD. In this way, such genes may be considered contributing susceptibility factors.

Such susceptibility factors may be the genes that have been identified by studies of the genetic architecture of ASD (see above), such as the neuroligins/neurorexins, the contactins, genes of the mTOR pathway (e.g. TSC1, TSC2 and AKT3), CYFIP1, SCN1A, EN2, SLC6A4, and BCKDK. The latter 2 genes have been implicated in epilepsy, while the mTOR pathway is involved in disorders such as tuberous sclerosis and in a hemimegalencephaly syndrome [Lee et al., 2012]. The 'classical' ASD genes of the neuroligin/neurorexin family and the contactins have thus far received most attention [Li et al., 2012; Peñagarikano and Geschwind, 2012].

To become valid targets for potential treatments of ASD, potential susceptibility genes need careful scrutiny. Features such as frequencies of mutations in patients versus the healthy individuals, predictions of the impact of a mutation on the structure and function of the encoded protein, and the extent of evolutionary conservation are not sufficient criteria. To determine whether a specific mutation is indeed pathogenic, functional validation of this mutation in a model organism is essential. For a transgenic organism to become a valid model to study the pathophysiology of ASD and potential treatments, it has to fulfill 3 validation criteria [Chadman et al., 2009; Nestler and Hyman, 2010]. First, such an animal model should be based on a known gene mutation, such as a loss of or a mutation in the gene under scrutiny (the so-called construct validity). Second, the animal should clearly exhibit demonstrable and ideally quantifiable phenotypes of human patients (the face validity). Third, the animal model should respond to treatments that have been shown to be effective in humans (predictive validity).

\section{Animal Models to Study Candidate Genes and to Find Clues for Novel Treatments}

\section{MECP}

The first animal model thus investigated was a mouse in which exon 3 of $M e c p 2$, which encodes 116 amino acids including most of the methyl-CpG-binding domain, was deleted [Chen et al., 2001]. This deletion created a mouse typified by stagnation of brain growth and a reduced neu- 
ronal cell size. This mouse replicates the human Rett syndrome (RTT, MIM 312750), which is a childhood neurological disorder and one of the most common causes of mental retardation in females and fulfilled all other validity requirements outlined above. IGF1 treatment extended the life span of the animals, improved locomotor function, ameliorated breathing patterns, reduced irregularities in heart rates, and increased brain size by apparently correcting defective synaptic maturation in the brain [Tropea et al., 2009]. The authors suggested IGF1 as a candidate for pharmacological treatment of RTT and potentially of other CNS disorders caused by delayed synapse maturation [Tropea et al., 2009]. In addition, female heterozygous null mutant mice Mecp $2^{+/-}$showed latency of cortically sourced components, which has been associated with language and developmental delay in autism [Liao et al., 2012]. These mice also exhibited gamma-band abnormalities that have recently been identified as an endophenotype of idiopathic autism. Since both these cortical abnormalities were demonstrated in these mice, the authors suggest a common cortical pathophysiology for RTT and ASD, and indicate that the Mecp $2^{+/-}$model may be useful for preclinical development targeting specific cortical processing abnormalities in RTT and potentially ASD [Liao et al., 2012]. In view of these results, female Mecp 2 heterozygous mutant mice represent a promising model to study potential treatments for both RTT and ASD.

\section{FMR 1 and CYFIP1}

The fragile $\mathrm{X}$ mental retardation syndrome (also known as the Martin-Bell syndrome, MIM 300624) is the most common X-linked form of mental retardation in males and also a frequent cause of infantile autism. In most males, the disorder is caused by the unstable expansion of a CGG repeat in the FMR1 gene (Xq27.3), often visible as a fragile site, leading to abnormal methylation, which results in suppression of FMR1 transcription, decreased protein levels in the brain and altered synaptic plasticity [Devys et al., 1993]. Given the preponderance of boys with ASD, but not fragile X mental retardation syndrome (see Introduction), the FMR1 gene may also be a likely contributor to ASD. A second potential overlap emerged after the unexpected finding that neuroligin-3 knockout mice exhibit the same disrupted heterosynaptic competition and metabotropic glutamate receptor-dependent synaptic plasticity as found in humans with the fragile X syndrome [Baudouin et al., 2012]. Re-expression of wild type neuroligin 3 rescued this phenotype in mice. This suggested that autism and the fragile $\mathrm{X}$ syndrome share defects in neuronal plasticity, which can be reverted even after brain development seemed to have been completed [Baudouin et al., 2012].

Recently, a family has been identified in which a hemizygous loss of part of region 15q11.2, containing the CYFIP1 gene, cosegregated with ASD [van der Zwaag et al., 2010]. A mouse with a hemizygous loss of the Cyfip1 gene also mimicked key aspects of the Fmrl knockout mouse [Bozdagi et al., 2012]. This mouse is of particular interest to autism research since a hemizygous loss of region 15q11.2, containing CYFIP1 and 3 other genes, was found to cosegregate with autism in a multiplex family [van der Zwaag et al., 2010]. In addition, in a cohort of 854 ASD patients, a single patient with PDD-NOS and mild intellectual delay carrying a $\mathrm{CNV}$, containing the SHANK2 gene and a second $\mathrm{CNV}$ covering region 15q11.2, had been identified [Leblond et al., 2012]. These 2 observations on CNVs involving CYFIP1 in humans indicate that this mouse fulfills 2 of the 3 requirements for being a valid model for autism: construct validity (hemizygous loss of Cyfip1) and face validity (replicating key phenotypes). This mouse is also of value for studying the hypothesis of Abrahams and Geschwind [2008] regarding the possible interaction between autosomal (CYFIP1 on human 15q11.2) and X-chromosomal (FMR1 on human Xq27.3) genes and its relevance for explaining the strong bias of ASD prevalence toward males. Finally, these findings highlight the phenotypic overlap between autism and some mental retardation syndromes.

\section{TSC1, SCN1A and BCKDK}

Further evidence for phenotypic overlap of autism with other disorders has been found in Tscl mutant mice [Tsai et al., 2012] and in Scn1a ${ }^{+/-}$mice [Han et al., 2012]. Both hemizygous and homozygous loss of Tsc1 in the Purkinje cells of the cerebellum of mice resulted in autistic-like behaviors, such as abnormal social interaction, repetitive behavior and vocalizations, and in decreased excitability of the Purkinje cells [Tsai et al., 2012]. Treatment of mutant mice with the mTOR inhibitor, rapamycin, prevented both pathological and behavioral defects. The findings with these mice demonstrate new roles for TSC1 in Purkinje cell function and allow defining a molecular basis for a contribution of the cerebellum to disorders such as autism [Tsai et al., 2012].

Mice with a hemizygous loss of Scn1a exhibit hyperactivity, stereotyped behaviors, social interaction deficits, and impaired context-dependent spatial memory [Han et al., 2012]. Although olfactory sensitivity was retained, novel food odors and social odors elicited aversive reactions in these mice. Interestingly, GABAergic neurotrans- 
mission was specifically impaired by $S c n 1 A$ hemizygosity, and selective deletion of $\mathrm{Na}(\mathrm{V}) 1.1$ channels in forebrain interneurons was sufficient to provoke these behavioral and cognitive impairments. Treatment with low-dose clonazepam, a positive allosteric modulator of GABA(A) receptors, completely rescued the abnormal social behaviors and deficits in fear memory in these mice. This indicates that $S c n 1 A$ haploinsuffiency in mice, which is intended to represent a mouse model of Dravet's syndrome, indeed involved impaired GABAergic neurotransmission and is the result of neuronal damage from recurrent seizures. These results demonstrate a critical role for $\mathrm{Na}(\mathrm{V}) 1.1$ channels in neuropsychiatric functions and provide a potential therapeutic strategy for cognitive deficit and autism-spectrum behaviors in patients with SCN1A-related Dravet's syndrome. Together, these mice corroborate genetic data indicating a contribution of TSC1 and SCN1A to autism and provide valid models to study the pathology and possible novel treatments of ASD.

In 2 families, homozygous loss-of-function mutations in the nuclear-encoded mitochondrial branched-chain keto acid dehydrogenase kinase gene (BCKDK) had been found [Novarino et al., 2012]. The patients presented with ASD, intellectual delay, seizures, or abnormalities in the electroencephalogram, and were part of consanguineous families. By homozygosity mapping, a region of identityby-descent containing the $B C K D K$ gene on chromosomal region $16 \mathrm{p} 11.2$ was identified. In the first family, a homozygous premature stop codon at amino acid position 156 and in the second family a homozygous frameshift mutation terminating the protein at amino acid 74 was detected. In these patients, the levels of $B C K D K$ mRNA were reduced, which is consistent with nonsense-mediated mRNA decay. No BCKDK protein was detected by Western blotting, confirming the null effect of the homozygous mutations. Bckdk knockout mice recapitulated both biochemical and neurobehavioral sequelae of the BCKDK mutations in humans. Supplementation of the diet of these mice with branched chain amino acids ameliorated their biochemical and behavioral phenotypes. In this way, the authors discovered a rare Mendelian cause of autism, which resulted from a metabolic disorder that can be reversed by dietary supplementation. Detection of $B C K D K$ mutations and subsequently lowered serum levels of branched chain amino acids may in these specific patients be enough to indicate treatment by dietary supplementation.

\section{Neuroligins}

Neuronal adhesion molecules, in particular neuroligins and neurexins, constitute the by far most studied clues toward a genetic etiology of autism. These genes gained attention after mutations in neuroligins 3 and 4 (NLGN3 in human region Xq13.1 and NLGN4 in human region Xp22, respectively) linking these genes to ASD [Jamain et al., 2003]. In 2 transgenic mouse lines, 2 distinct Nlgn 3 mutations were introduced [Etherton et al., 2011a, 2011b]. The Nlgn3 R704C mutation did not significantly alter synapse formation but impaired synapse function and caused a major and selective decrease in AMPA receptor-mediated synaptic transmission in pyramidal neurons of the hippocampus, without affecting NMDA (N-methyl-D-aspartate) or GABA receptor-mediated synaptic transmission and without detectably altering presynaptic neurotransmitter release [Etherton et al., 2011a]. In contrast, a R451C knockin mouse exhibited modestly impaired social behaviors and increased synaptic inhibition in the somatosensory cortex [Etherton et al., 2011b]. These behavioral changes may be due to a shift of synaptic transmission to inhibition. In addition, the R451C mutant mice exhibited increased synaptic inhibition in the somatosensory cortex. In the hippocampus, however, the R451C mutation produced a strikingly different phenotype. In the hippocampal CA1 region, the R451C mutation caused an $~ 1.5$-fold increase in AMPA receptor-mediated excitatory synaptic transmission, strongly altered the kinetics of NMDA receptor-mediated synaptic responses, induced an approximately 2 -fold upregulation of NMDA receptors containing NR2B subunits, and enhanced long-term potentiation almost 2 -fold. Nlgn3 knockout mice did not exhibit any of these changes. Quantitative light microscopy and electron microscopy revealed that the Nlgn3 R451C mutation increased dendritic branching and altered the structure of synapses in the stratum radiatum of the hippocampus. Thus, in Nlgn3 R451C mutant mice, a single point mutation in a synaptic cell adhesion molecule causes context-dependent changes in synaptic transmission. These changes are consistent with the broad impact of this mutation on murine and human behaviors. The distinct findings with Nlgn 3 knockout mice and the 2 point mutations indicated that NLGN3 controls excitatory and inhibitory synapse properties of the brain in a region- and circuit-specific manner [Etherton et al., 2011b].

Neuroligin 1 (NLGN1 in human region 3q26.3) mutant mice were also studied regarding autism- and mental retardation-relevant behavioral tasks [Blundell et al., 2010]. Nlgn1 knockout mice display deficits in spatial learning and memory that correlate with impaired hippocampal long-term potentiation. In addition, these mice exhibit a dramatic increase in repetitive, stereotyped 
grooming behavior, akin to repetitive self-centered behavior in ASD. This repetitive grooming abnormality in mice is associated with a reduced NMDA/AMPA ratio at corticostriatal synapses. The increased repetitive grooming phenotype was rescued in adult mice by administration of the NMDA receptor partial coagonist d-cycloserine. These results are consistent with a role of synaptic cell-adhesion molecules in general in autism and implicate reduced excitatory synaptic transmission as a potential mechanism and treatment target for repetitive behavioral abnormalities [Blundell et al., 2010].

In contrast to mice with Nlgn3 point mutations (see above), mutant mice lacking the mouse Nlgn2 gene displayed decreased inhibitory synaptic function and an apparent decrease in the number of inhibitory synaptic puncta [Blundell et al., 2009]. However, as shown by electron microscopy, the number of symmetric synapses is unaltered, suggesting that Nlgn2 deletion impairs the function of inhibitory synapses without decreasing their numbers. This decrease in inhibitory synaptic function in Nlgn2-deficient mice correlated with a discrete behavioral phenotype. These mice showed a marked increase in anxiety-like behavior, a decrease in pain sensitivity and a slight decrease in motor co-ordination. These observations confirm that NLGN2 modulates inhibitory synaptic function and demonstrates that deletion of Nlgn2 can lead to a selective, autism-like behavioral phenotype [Blundell et al., 2009]. Knocking out the eukaryotic translation initiation factor $4 \mathrm{E}$-binding protein 2 (4E-BP2), which is part of the mTOR pathway, provokes increased translation of neuroligins and subsequent dysregulation of the excitation-inhibition balance in the mouse brain [Gkogkas et al., 2013]. For a comprehensive review of the currently available neuroligin mouse models, the reader is referred to Xu et al. [2012].

\section{Neurexins}

The neurexins represent a class of presynaptic cell-adhesion molecules, which form transsynaptic complexes with postsynaptic neuroligins [Toro et al., 2010]. Upon overexpression in nonneuronal cells, neurexins induce formation of postsynaptic specializations in cocultured neurons, which suggests that neurexins are synaptogenic [Zhang et al., 2010]. When overexpressed in neurons, however, neurexins do not increase synaptic density, but instead suppressed GABAergic synaptic transmission without decreasing numbers of GABAergic synapses. This effect was mediated by all subtypes of neurexins tested in a cell-autonomous and neuroligin-independent manner. Remarkably, addition of recombinant neurexin to cultured neurons at submicromolar concentrations in- duced the same suppression of GABAergic synaptic transmission as neurexin overexpression. In addition, neurexins bind directly and stoichiometrically to GABA(A) receptors, suggesting that neurexins decrease GABAergic synaptic responses by interacting with GABA(A) receptors. These findings indicate that presynaptic neurexins act directly on postsynaptic GABA(A) receptors. This feature may contribute to regulate the excitatory/inhibitory balance in brain and if disturbed, may precipitate behavioral phenotypes as seen in ASD.

\section{CNTNAP2}

The most studied neurexin is contactin associated protein 2, CNTNAP2, a 2.3-Mb gene located in human region 7q36.1, which has been implicated in ASD [Poot et al., 2010a, 2011; Peñagarikano and Geschwind, 2012] and related to neurodevelopmental disorders [Vernes et al., 2008; Newbury et al., 2010; Peñagarikano and Geschwind, 2012]. Mice in which the Cntnap2 gene has been deleted show deficits in the 3 core ASD behavioral domains, as well as hyperactivity and epileptic seizures, as have been reported in humans with CNTNAP2 mutations [Peñagarikano et al., 2011]. Neuropathological and physiological analyses of these mice before the onset of seizures reveal abnormal neuronal migration, lowered numbers of interneurons and abnormal neuronal network activity. Treatment with risperidone ameliorates the repetitive behaviors in the mutant mice. This study indicates a functional role for CNTNAP2 in brain development and provides a new model for research into the pathology, and potential and novel therapeutic approaches in ASD.

\section{SHANK2}

Studying SHANK2, a scaffolding protein located in the postsynaptic density of glutamatergic synapses, Leblond et al. [2012] found evidence for a multiple-hit model of ASD in which SHANK2 interacts with 'usual suspects' such as CHRNA7 and CYFIP1. A limitation of such studies of cohorts of patients is that one cannot attribute specific phenotypes to a specific gene mutation, if several genes were affected at once in a single individual. This . limitation can be overcome by generating single gene mutation model organisms. Shank2-mutant (i.e. Shank2 ${ }^{-/-}$) mice carrying a CNV identical to the ASD-associated microdeletion in the human SHANK2 gene exhibit ASDlike behaviors, such as reduced social interaction, reduced social communication by ultrasonic vocalizations and repetitive jumping [Won et al., 2012]. These mice show a marked decrease in NMDA glutamate receptor (NMDAR) function. Direct stimulation of NMDARs 
Fig. 1. CNVs of the family with a hemizygous loss of a part of 11q22.1 (a) and 2 families with an inherited loss (family B) and a de novo gain (family C) of a part of 3p26 (b). The vertical bars above and below the symbols for the individuals in the pedigrees indicate their SNP haplotypes for the concerned chromosomal regions. The bars below the genomic maps indicate the nature and extent of the CNV. Red bar = Hemizygous loss; green bar = gain; n.a. = not available.

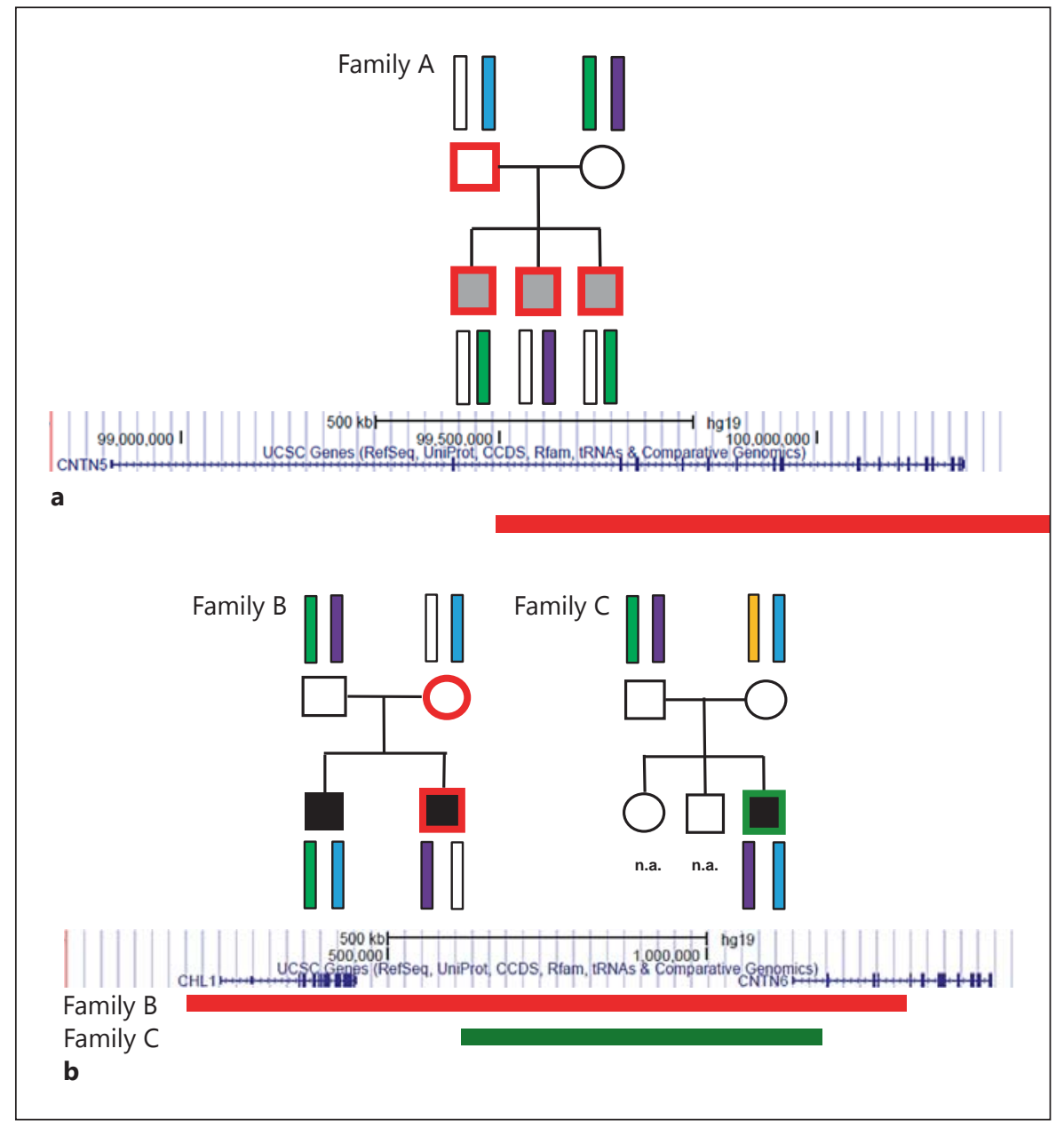

with d-cycloserine, a partial agonist of NMDARs, normalizes NMDAR function and improves social interaction in Shank $2^{-/-}$mice. Treatment of Shank $2^{-/-}$mice with a positive allosteric modulator of metabotropic glutamate receptor 5 (mGluR5) also normalizes NMDAR function and markedly enhances social interaction. These results suggest that reduced NMDAR function, resulting from deletion of both alleles of Shank2 in mice, may contribute to the development of ASD-like phenotypes and that mGluR modulation of NMDARs may offer a potential strategy to treat ASD [Won et al., 2012].

In an independent transgenic mouse model, Schmeisser et al. [2012] showed that deletion of both Shank2 alleles results in an early, brain region-specific upregulation of ionotropic glutamate receptors at the synapses and increased levels of ProSAP2/Shank3. Shank $2^{-/-}$mutants exhibit fewer dendritic spines and show reduced basal synaptic transmission, a reduced frequency of miniature excitatory postsynaptic currents and enhanced N-methyl-d-aspartate receptor-mediated excitatory currents at the physiological level. Mutants are extremely hyperactive and display profound autistic-like behavioral alterations including repetitive grooming as well as abnormalities in vocal and social behaviors. Comparing the data on ProSAP1/Shank $2^{-/-}$mutants with ProSAP2/Shank $3 \alpha \beta^{-/-}$mice, the authors observed that different abnormalities in synaptic glutamate receptor expression can cause distinct alterations in social interactions and communication. Therefore, the authors propose that therapies for ASD should be carefully matched to the underlying synaptopathic phenotype, which requires high-resolution genomic analysis of each individual patient.

\section{Contactins}

In a recent study of a cohort of ASD patients, a transmitted loss of a part of CNTN5 has been found (fig. 1a), which indicates that this particular CNV is by itself suf- 
ficient to cause autism in the 3 affected children in this family [van Daalen et al., 2011]. This loss could either lead to transcription of a truncated part of CNTN5 (exons 1 and 2) or may, by nonsense mediated decay, not produce any mRNA. In either case, the loss would be pathogenic by leading to haploinsufficiency for CNTN5 [Poot et al., 2011]. Since the 3 affected boys inherited different copies of chromosome 11 from their mother (vertical bars in fig. 1a), unmasking of a putative heterozygous mutation on a maternal chromosome by the paternally transmitted deletion is contradicted [Hochstenbach et al., 2011].

The CNTN6 gene in chromosomal region 3p26 was affected by an inherited large loss also encompassing the CH1L gene (family B in fig. $1 \mathrm{~b}$ ) and by a de novo gain of the promoter region and exon 1 in family $\mathrm{C}$ (fig. 1b). The latter turned out to be a tandem duplication [van Daalen et al., 2011], which may be pathogenic by disrupting the reading frame of CNTN6, similar to findings of $\mathrm{MCPH1}$ in ASD [Ozgen et al., 2009]. The hemizygous loss of $C H 1 L$ and part of CNTN6 in family B, in contrast, is by itself not sufficient to be pathogenic, since in this family there is a second affected, older brother without the loss (fig. 1b). Both brothers inherited distinct chromosomes 3 from their unaffected father (family B in fig. 1b). This contradicts unmasking of a putative heterozygous mutation on a paternal chromosome by the maternally transmitted deletion [Hochstenbach et al., 2011].

Even before the human data were published, Cntn 5 had been subjected to intense study in mouse models [ $\mathrm{Li}$ et al., 2003; Toyoshima et al., 2009; Shimoda et al., 2012]. Li et al. [2003] ablated a part of the Cntn5/NB-2 gene by inserting the tau-LacZ gene in the NB-2 locus of the mouse genome. $L a c Z$ expression patterns in heterozygous mice revealed that NB-2 is preferentially expressed in the auditory pathways. During an audiogenic seizure test, mutant mice exhibited a lower incidence of wild, explorative running. With c-Fos immunohistochemistry, the authors demonstrated that neural excitability induced by the audiogenic seizure test in the mutant mice was prominently attenuated in both the dorsal and external cortices of the inferior colliculus. These results indicate that a lack of $C n t n 5 / N B-2$ expression impaired the neuronal activity in the auditory system in the mouse, which has been also observed in some human ASD patients. By demonstrating strong Cntn5/NB-2 expression in the ventral cochlear nucleus, ventral acoustic stria, lateral and medial superior olvary complex, superior paraolivary nucleus, medial nucleus of the trapezoid body, ventrolateral lemniscus, and central nucleus of the inferior colliculus, Toyoshima et al. [2009] further supported a role for this gene in the auditory pathway. In situ hybridization combined with immunohistochemistry indicated that CNTN5/NB-2 is expressed only in neurons, where it colocalized with glutamatergic elements in the neuropil and the calyces of Held but not with glycinergic or GABAergic elements. Cntn5/NB-2 expression in the superior olvary complex was first detected at embryonic day (E)19, reached a maximum level at postnatal day $(\mathrm{P}) 7$ and declined thereafter. Immunolabeling with VGLUT1 and VGLUT2 markers mature and premature glutamatergic synapses, respectively. In combination with CNTN5/NB2 , immunolabeling revealed that CNTN5/NB-2 is expressed at glutamatergic synapses. These findings suggest that CNTN5/NB-2 plays a key role in maturation of glutamatergic synapses in the brainstem during the final stages of auditory development. More recently, it has been shown that the CNTN5/NB-2 protein colocalizes with amyloid precursor-like protein 1 in the presynaptic membrane [Shimoda et al., 2012]. This is commensurate with a possible involvement of CNTN5/NB-2 in governing neurite outgrowth and development, which, if altered, may eventually entail autistic phenotypes.

\section{Engrailed-2}

The engrailed-2 gene (EN2) encodes a homeobox transcription factor, which functions as a patterning gene in the early development and connectivity of rodent hindbrain and cerebellum, and regulates neurogenesis and development of monoaminergic pathways. Neuroanatomical expression profiling of En2 wild type mice demonstrated that EN2 is expressed in adult brain structures including the somatosensory cortex, hippocampus, striatum, thalamus, hypothalamus, and brainstem [Brielmaier et al., 2012]. En2 null mice, in contrast, exhibited deficits in reciprocal social interactions as juveniles and adults, and absence of sociability in adults, replicated in 2 independent cohorts. Fear conditioning and water maze learning were also impaired in En 2 knockout mice. However, no genotype differences were found on measures of ultrasonic vocalizations in social contexts, and no stereotyped or repetitive behaviors were observed. Developmental milestones, general health, olfactory abilities, exploratory locomotor activity, anxiety-like behaviors, and pain responses did not differ between wild type and knockout mice. While these knockout mice represent a model with construct and face validity, no response to possible treatments has as yet been reported. Yet, these mice represent a model to test treatments aiming at ameliorating deficits in social interaction in ASD patients and possibly other conditions. 
SLC6A4

Mutations in the 5-hydroxytryptamine transporter gene (SLC6A4) have been identified in children with ASD. Transgenic mice expressing the most common of these variants, SERT Ala56, exhibit elevated, p38 MAPKdependent transporter phosphorylation, enhanced 5-HT clearance rates and hyperserotonemia [Veenstra-VanderWeele et al., 2012]. These SERT Ala56 mice display alterations in social function, communication and repetitive behavior. These findings provide support for the hypothesis that altered 5-hydroxytryptamine homeostasis may impair a risk for ASD-related phenotypes and may provide a model with construct and face validity that can be used to further analyze the pathogenesis of ASD and potentially novel treatment modalities.

\section{Clinical Implications and Novel Clues for the Study and Treatment of Autism Spectrum Disorders}

Genotyping of several large cohorts of ASD patients have revealed a remarkably complex genomic architecture of this disorder. Although common variants, mutations in genes for Mendelian disease and structural chromosomal abnormalities have frequently been detected, they may, even in aggregate, account for only a small proportion of the underlying genetic causes. The majority of ASD cases may have resulted from a pathogenic convergence of multiple variants [Zhao et al., 2007; Nishiyama et al., 2009]. Such a polygenic model, in which multiple CNVs and SNVs function together in an additive or an epistatic manner [Cook and Scherer, 2008; Girirajan and Eichler, 2010; Poot et al., 2011; Leblond et al., 2012], appears at this stage the most plausible for the majority of ASD cases.

Single gene transgenic mouse studies generated numerous highly promising clues (table 1). Such models are limited since they can interrogate only a single gene at a time. In addition, many of these genes have been implicated in multiple disorders. The latter feature, paradoxically, allows taking into account that many ASD patients suffer from comorbidities such as intellectual delay and epilepsy. For instance, a Nlgn 3 knockout mouse recapitulated disrupted heterosynaptic competition and disruption of metabotropic glutamate receptor-dependent synapses as found in humans with the fragile $\mathrm{X}$ syndrome [Baudouin et al., 2012]. This further underscores the phenotypic overlap between ASD and the fragile X syndrome and other neurobehavioral disorders, and makes these mice an even more valuable resource for neuroanatomi- cal, pathological and behavioral studies geared at developing novel therapeutic approaches for ASD and potentially other disorders. Although this mouse model thus far represents a single finding, these data suggest that modulation of glutamate receptor functions may indeed become a possible avenue for pharmacologic intervention in selected patients with either ASD or the fragile X syndrome.

The recent flurry of transgenic mice has also provided early key insights in the neuroanatomical and pathological alterations accompanying ASD, yet the reported data are difficult to translate from one mouse to another. For these models, to fully unfold their potential, gaps in behavioral and neuroanatomical phenotyping have to be filled (see also table 1, which summarizes the most salient features of some of the mice reported thus far). Eventually, a tool of standardized phenotyping akin to the tools used with human patients, such as ADI-R, ADOS-G, DSM, and SRS, should be applied to all transgenic models. Even at this early point in time, several common themes begin to emerge. For instance, Fmr1, Shank2 and Cntn 5 knockout mice exhibit alterations of glutamate receptors at the synapse, albeit in distinct regions of the brain. If corroborated by further studies, these findings may suggest that only a limited number of neuroanatomical mechanisms may be involved in the causation of a disorder as complex as ASD.

Recently Nlgn4 null mice have been extensively phenotyped with the aim to develop an 'Autism Severity Score' [El-Kordi et al., 2012]. Interestingly, male and female monogenic mutant mice exhibited a similar pattern of coordinated behavioral impairments, albeit females were less severely affected than males. In this way, comprehensive phenotyping of these monogenic mutant mice not only served as a point-of-entry into a complex disorder, it also indicates that some domains of impaired functioning in ASD share a common genetic and neurobiological origin. In this case, complete loss of Nlgn 4 reduced social interaction and ultrasonic communication, whereas aggressiveness, nest building and self-grooming were not affected. Thus, reduced social interaction and ultrasonic communication may be a 'Neuroligin biology-related' ASD endophenotype. It is conceivable that identification of such endophentypes in ASD transgenic mice may serve as a proxy for underlying pathways of perturbed biological function and even genetic mutation. The finding that male mice are more severely affected than their female littermates is commensurate with the finding that male patients are generally more severely affected than females. Taken together, the findings of this study [ElKordi et al., 2012] underscore the need for comprehen- 
Table 1. Some recently published mouse models for ASD

\begin{tabular}{|c|c|c|c|c|c|}
\hline Gene & Localization & Major comorbidities & Major brain pathologies & $\begin{array}{l}\text { Treatment } \\
\text { options }\end{array}$ & References \\
\hline$M E C P 2$ & $\mathrm{Xq} 28$ & Rett syndrome (MIM: 312750) & $\begin{array}{l}\text { brain size, synaptic transmis- } \\
\text { sion }\end{array}$ & IGF1 & $\begin{array}{l}\text { Tropea et al., 2009; } \\
\text { Liao et al., } 2012\end{array}$ \\
\hline FMR1 & $\mathrm{Xq} 27.3$ & $\begin{array}{l}\text { fragile X (Martin-Bell) syndrome } \\
\text { (MIM: 300624) }\end{array}$ & $\begin{array}{l}\text { heterosynaptic transmission, } \\
\text { glutamate receptor-dependent } \\
\text { synaptic plasticity }\end{array}$ & no & Bozdagi et al., 2012 \\
\hline TSC1 & $9 q 34.13$ & tuberous sclerosis (MIM: 191100) & & rapamycin & Tsai et al., 2012 \\
\hline SCN1A & $2 \mathrm{q} 24.3$ & $\begin{array}{l}\text { Dravet syndrome: severe myoclonic } \\
\text { epilepsy, childhood epileptic tonic } \\
\text { clonic seizures (MIM: 607208); } \\
\text { familial hemiplegic migraine (MIM: } \\
\text { 609634); familial febrile convulsions } \\
\text { 3A (MIM: 604403) }\end{array}$ & & $\begin{array}{l}\text { low-dose } \\
\text { clonazepam }\end{array}$ & Han et al., 2012 \\
\hline NLGN3 & $\mathrm{Xq13.1}$ & & $\begin{array}{l}\text { heterosynaptic transmission, } \\
\text { glutamate receptor-dependent } \\
\text { synaptic plasticity, increase or } \\
\text { decrease in AMPA-mediated } \\
\text { synaptic transmission, altered } \\
\text { synaptic branching }\end{array}$ & no & $\begin{array}{l}\text { Etherton et al., 2011a; } \\
\text { Etherton et al., 2011b; } \\
\text { Baudouin et al., } 2012\end{array}$ \\
\hline NLGN1 & $3 \mathrm{p} 26.1$ & & $\begin{array}{l}\text { impaired hippocampal long- } \\
\text { term potentiation, reduced } \\
\text { NMDA/AMPA ratio at } \\
\text { corticostriatal synapses }\end{array}$ & no & Blundell et al., 2010 \\
\hline SHANK2 & $11 \mathrm{q} 13.3$ & & $\begin{array}{l}\text { decrease in NMDA-glutamate } \\
\text { receptor (NMDAR) function; } \\
\text { brain region-specific upregula- } \\
\text { tion of ionotropic glutamate } \\
\text { receptors at synapse, increased } \\
\text { levels of ProSAP2/Shank3 }\end{array}$ & D-cycloserine & $\begin{array}{l}\text { Schmeisser et al., 2012; } \\
\text { Won et al., } 2012\end{array}$ \\
\hline CNTN5 & $11 q 22.1$ & & $\begin{array}{l}\text { altered maturation of gluta- } \\
\text { matergic synapses in the } \\
\text { brainstem }\end{array}$ & no & $\begin{array}{l}\text { Li et al., 2003; } \\
\text { Toyoshima et al., 2009; } \\
\text { Shimoda et al., } 2012\end{array}$ \\
\hline EN2 & $7 q 36.3$ & & not investigated & no & Brielmaier et al., 2012 \\
\hline SLC6A4 & $17 q 11.2$ & & $\begin{array}{l}\text { elevated 5-HT transporter } \\
\text { phosphorylation, enhanced } \\
\text { 5-HT clearance and hyper- } \\
\text { serotonemia }\end{array}$ & no & $\begin{array}{l}\text { Veenstra-VanderWeele } \\
\text { et al., } 2012\end{array}$ \\
\hline
\end{tabular}


sive phenotyping and suggest that single locus mutant mice may help us to resolve the neuro- and behavioral biology and the genetic conundrums of ASD.

These neurobiological and behavioral changes in transgenic mice need to be examined more profoundly, since they may provide us with potential clues for pharmacologic intervention. Such targeted interventions may prove fruitful for not only ASD but also for patients with the fragile X syndrome and even less clearly defined forms of intellectual delay [Poot et al., 2010b]. Maybe, the most surprising outcome of transgenic mice studies thus far is the finding that some of the phenotypes of Mecp 2 knockout mice can be ameliorated by administration of IGF1 [Tropea et al., 2009]. This not only suggests a higher degree of brain plasticity than thus far appreciated but also suggests that ASD patients may still be amenable to treatment options during, or even after, puberty. Thus, IGF1 treatment may lead to dampening of adverse interactional phenotypes to such an extent that some ASD patients may become amenable to behavioral interventions.

Although studies of monogenic mutant mice begin to provide novel insights into the underlying neuro- and behavioral biology of ASD, they may not substitute the need for comprehensive phenotyping of each individual ASD patient. A recent study by Lim et al. [2013] has shown that biallelic mutations in a single gene are extremely rare among ASD patients. To the contrary, highly penetrant mutations and CNVs, exclusively associated with ASD, may be extremely rare or even nonexistent [Yu et al., 2013]. A leading to partial loss of gene function may be a common theme in ASD. These findings further corroborate the current model of an extremely complex genetic architecture and genetic heterogeneity underlying ASD [Yu et al., 2013]. As Schmeisser et al. [2012] have pointed out, proposals for possible therapies for ASD should be carefully matched to the underlying synaptopathic phenotype, which requires high-resolution genomic analysis. The latter should encompass high-resolution CNV analysis combined with either exome sequencing or complete sequencing of candidate regions. This approach may, in addition to finding variants in protein-coding sequences, also uncover regulatory variations and epigenetic mechanisms [Poot et al., 2011]. The eventual goal of this screening approach is to obtain a comprehensive overview of all variants that may contribute to the phenotype(s) of each individual patient in order to classify his or her genotype in terms of perturbed biological mechanisms.

Such combined pharmacological and behavioral approaches may not represent true causal therapies, but may push the ASD patient back into the 'normal distribution' of the general population with respect to, for instance, social interaction. Although the magic 'anti-ASD pill' may not become available soon, comprehensive genotyping of individual patients and siblings combined with the novel insights generated from the transgenic animal studies may eventually provide us with personalized treatment options for ASD patients. Eventually, autism may indeed become a phenotypically heterogeneous group of disorders ('autisms') caused by combinations of changes in multiple possible candidate genes [Toro et al., 2010] being different in each patient and requiring for each individual a combination of distinct, individuallytailored treatment(s).

\section{Acknowledgement}

This autism research of the author has been supported by a grant from the Hersenstichting (Netherlands Foundation for Brain Research) grant No. 2008(1).34.

\section{References}

-Abrahams BS, Geschwind DH: Advances in autism genetics: on the threshold of a new neurobiology. Nat Rev Genet 9:341-355 (2008).

American Psychiatric Association: Diagnostic and Statistical Manual of Mental Disorders (American Psychatric Publishing, Arlington 2000).

-Anney R, Klei L, Pinto D, Almeida J, Bacchelli E, et al: Individual common variants exert weak effects on the risk for autism spectrum disorders. Hum Mol Genet 21:4781-4792 (2012).
Autism Genome Project Consortium, Szatmari P, Paterson AD, Zwaigenbaum L, Roberts W, et al: Mapping autism risk loci using genetic linkage and chromosomal rearrangements. Nat Genet 39:319-328 (2007).

Bailey A, Le Couteur A, Gottesman I, Bolton P, Simonoff E, et al: Autism as a strongly genetic disorder: evidence from a British twin study. Psychol Med 25:63-77 (1995).

- Baudouin SJ, Gaudias J, Gerharz S, Hatstatt L, Zhou K, et al: Shared synaptic pathophysiology in syndromic and nonsyndromic rodent models of autism. Science 338:128-132 (2012).
Blundell J, Tabuchi K, Bolliger MF, Blaiss CA, Brose N, et al: Increased anxiety-like behavior in mice lacking the inhibitory synapse cell adhesion molecule neuroligin 2. Genes Brain Behav 8:114-126 (2009).

Blundell J, Blaiss CA, Etherton MR, Espinosa F, Tabuchi K, et al: Neuroligin-1 deletion results in impaired spatial memory and increased repetitive behavior. J Neurosci 30:2115-2129 (2010).

Bozdagi O, Sakurai T, Dorr N, Pilorge M, Takahashi N, Buxbaum JD: Haploinsufficiency of Cyfip1 produces fragile X-Like phenotypes in mice. PLoS One 7:e.42422 (2012). 
-Brielmaier J, Matteson PG, Silverman JL, Senerth JM, Kelly S, et al: Autism-relevant social abnormalities and cognitive deficits in engrailed-2 knockout mice. PLoS One 7:e40914 (2012).

- Bucan M, Abrahams BS, Wang K, Glessner JT, Herman EI, et al.: Genome-wide analyses of exonic copy number variants in a familybased study point to novel autism susceptibility genes. PLoS Genet 5:e1000536 (2009).

-Buizer-Voskamp JE, Laan W, Staal WG, Hennekam EA, Aukes MF, et al: Paternal age and psychiatric disorders: findings from a Dutch population registry. Schizophr Res 129:128132 (2011).

-Chadman KK, Yang M, Crawley JN: Criteria for validating mouse models of psychiatric diseases. Am J Med Genet B Neuropsychiatr Genet 150B:1-11 (2009).

-Chen RZ, Akbarian S, Tudor M, Jaenisch R: Deficiency of methyl-CpG binding protein-2 in CNS neurons results in a Rett-like phenotype in mice. Nat Genet 27:327-331 (2001).

-Cook EH Jr, Scherer SW: Copy-number variations associated with neuropsychiatric conditions. Nature 455:919-923 (2008).

-Cuscó I, Medrano A, Gener B, Vilardell M, Gallastegui F, et al: Autism-specific copy number variants further implicate the phosphatidylinositol signaling pathway and the glutamatergic synapse in the etiology of the disorder. Hum Mol Genet 18:1795-1804 (2009).

-Devlin B, Scherer SW: Genetic architecture in autism spectrum disorder. Curr Opin Genet Dev 22:229-237 (2012).

-Devys D, Lutz Y, Rouyer N, Bellocq JP, Mandel JL: The FMR-1 protein is cytoplasmic, most abundant in neurons and appears normal in carriers of a fragile X premutation. Nat Genet 4:335-340 (1993).

-El-Kordi A, Winkler D, Hammerschmidt K, Kästner A, Krueger D, et al: Development of an autism severity score for mice using Nlgn4 null mutants as a construct-valid model of heritable monogenic autism. Behav Brain Res pii: S0166-4328(12)00738-3 (2012).

Etherton MR, Tabuchi K, Sharma M, Ko J, Südhof TC: An autism-associated point mutation in the neuroligin cytoplasmic tail selectively impairs AMPA receptor-mediated synaptic transmission in hippocampus. EMBO J 30: 2908-2919 (2011a).

Etherton M, Földy C, Sharma M, Tabuchi K, Liu $\mathrm{X}$, et al: Autism-linked neuroligin-3 R451C mutation differentially alters hippocampal and cortical synaptic function. Proc Natl Acad Sci USA 108:13764-13769 (2011b).

Fombonne E: A wrinkle in time: from early signs to a diagnosis of autism. J Am Acad Child Adolesc Psychiatry 48:463-464 (2009).

Freitag CM: The genetics of autistic disorders and its clinical relevance: a review of the literature. Mol Psychiatry 12:2-22 (2007).

-Gillberg C, Coleman M: Autism and medical disorders: a review of the literature. Dev Med Child Neurol 38:191-202 (1996).
Girirajan S, Eichler EE: Phenotypic variability and genetic susceptibility to genomic disorders. Hum Mol Genet 19:R176-187 (2010).

-Girirajan S, Rosenfeld JA, Coe BP, Parikh S, Friedman N, et al: Phenotypic heterogeneity of genomic disorders and rare copy-number variants. N Engl J Med 367:1321-1331 (2012).

Gkogkas CG, Khoutorsky A, Ran I, Rampakakis E, Nevarko T, et al: Autism-related deficits via dysregulated eIF4E-dependent translational control. Nature 493:371-377 (2013).

Glessner JT, Wang K, Cai G, Korvatska O, Kim $\mathrm{CE}$, et al: Autism genome-wide copy number variation reveals ubiquitin and neuronal genes. Nature 459:569-573 (2009).

Hallmayer J, Cleveland S, Torres A, Phillips J, Cohen $\mathrm{B}$, et al: Genetic heritability and shared environmental factors among twin pairs with autism. Arch Gen Psychiatry 2011;68:10951102.

Han S, Tai C, Westenbroek RE, Yu FH, Cheah CS, et al: Autistic-like behaviour in Scn1a+/- mice and rescue by enhanced GABA-mediated neurotransmission. Nature 489:385-390 (2012).

Hochstenbach R, Buizer-Voskamp JE, Vorstman JA, Ophoff RA: Genome arrays for the detection of copy number variations in idiopathic mental retardation, idiopathic generalized epilepsy and neuropsychiatric disorders: lessons for diagnostic workflow and research. Cytogenet Genome Res 135:174-202 (2011).

-Holt R, Barnby G, Maestrini E, Bacchelli E, Brocklebank $\mathrm{D}$, et al: Linkage and candidate gene studies of autism spectrum disorders in European populations. Eur J Hum Genet 18:10131019 (2010).

Iossifov I, Ronemus M, Levy D, Wang Z, Hakker I, et al: De novo gene disruptions in children on the autistic spectrum. Neuron 74:285-299 (2012).

Itsara A, Cooper GM, Baker C, Girirajan S, Li J, et al: Population analysis of large copy number variants and hotspots of human genetic disease. Am J Hum Genet 84:148-161 (2009).

-Jacquemont ML, Sanlaville D, Redon R, Raoul O, Cormier-Daire V, et al: Array-based comparative genomic hybridisation identifies high frequency of cryptic chromosomal rearrangements in patients with syndromic autism spectrum disorders. J Med Genet 43:843-849 (2006).

Jamain S, Quach H, Betancur C, Råstam M, Colineaux $\mathrm{C}$, et al: Mutations of the X-linked genes encoding neuroligins NLGN3 and NLGN4 are associated with autism. Nat Genet 34:27-29 (2003).

Kong A, Frigge ML, Masson G, Besenbacher S, Sulem P, et al: Rate of de novo mutations and the importance of father's age to disease risk. Nature 488:471-475 (2012).

Leblond CS, Heinrich J, Delorme R, Proepper C, Betancur C, et al: Genetic and functional analyses of SHANK2 mutations suggest a multiple hit model of autism spectrum disorders. PLoS Genet 8:e1002521 (2012).
Le Couteur A, Bailey A, Goode S, Pickles A, Robertson S, et al: A broader phenotype of autism: the clinical spectrum in twins. J Child Psychol and Psychiatry 37:785-801 (1996).

Lee JH, Huynh M, Silhavy JL, Kim S, Dixon-Salazar T, et al: De novo somatic mutations in components of the PI3K-AKT3-mTOR pathway cause hemimegalencephaly. Nat Genet 44:941-945 (2012).

Li H, Takeda Y, Niki H, Ogawa J, Kobayashi S, et al: Aberrant responses to acoustic stimuli in mice deficient for neural recognition molecule NB-2. Eur J Neurosci 17:929-936 (2003).

Li X, Zou H, Brown WT: Genes associated with autism spectrum disorder. Brain Res Bull 88: 543-552 (2012).

Liao W, Gandal MJ, Ehrlichman RS, Siegel SJ, Carlson GC: $\mathrm{MeCP} 2+/-$ mouse model of RTT reproduces auditory phenotypes associated with Rett syndrome and replicate select EEG endophenotypes of autism spectrum disorder. Neurobiol Dis 46:88-92 (2012).

- Lim ET, Raychaudhuri S, Sanders SJ, Stevens C, Sabo A, et al: Rare complete knockouts in humans: population distribution and significant role in autism spectrum disorders. Neuron 77 : 235-242 (2013).

Ma D, Salyakina D, Jaworski JM, Konidari I, Whitehead PL, et al: A genome-wide association study of autism reveals a common novel risk locus at 5p14.1. Ann Hum Genet 73:263273 (2009).

Magnée MJ, Kahn RS, Cahn W, Kemner C: More prolonged brain activity related to gaze cueing in schizophrenia. Clin Neurophysiol 122: 506-511 (2011).

Manolio TA, Collins FS, Cox NJ, Goldstein DB, Hindorff LA, et al: Finding the missing heritability of complex diseases. Nature 461:747753 (2009).

- Marshall CR, Noor A, Vincent JB, Lionel AC, Feuk L, et al: Structural variation of chromosomes in autism spectrum disorder. Am J Hum Genet 82:477-488 (2008)

Neale BM, Kou Y, Liu L, Ma'ayan A, Samocha KE, et al: Patterns and rates of exonic de novo mutations in autism spectrum disorders. Nature 485:242-245 (2012).

Nestler EJ, Hyman SE: Animal models of neuropsychiatric disorders. Nat Neurosci 13:11611169 (2010).

Newbury DF, Fisher SE, Monaco AP: Recent advances in the genetics of language impairment. Genome Med 2:6 (2010).

- Newschaffer CJ, Croen LA, Daniels J, Giarelli E, Grether JK, et al: The epidemiology of autism spectrum disorders. Annu Rev Public Health 28:235-258 (2007)

- Nishiyama T, Notohara M, Sumi S, Takami S, Kishino H: Major contribution of dominant inheritance to autism spectrum disorders (ASDs) in population-based families. J Hum Genet 54:721-726 (2009). 
Novarino G, El-Fishawy P, Kayserili H, Meguid Sanders SJ, Murtha MT, Gupta AR, Murdoch JD, NA, Scott EM, et al: Mutations in BCKDkinase lead to a potentially treatable form of autism with epilepsy. Science 338:394-397 (2012).

O'Roak BJ, Vives L, Girirajan S, Karakoc E, Krumm N, et al: Sporadic autism exomes reveal a highly interconnected protein network of de novo mutations. Nature 485:246-250 (2012).

-Ozgen HM, van Daalen E, Bolton PF, Maloney VK, Huang S, et al: Copy number changes of the microcephalin 1 gene $(M C P H 1)$ in patients with autism spectrum disorders. Clin Genet 76:348-356 (2009).

$\checkmark$ Peñagarikano O, Abrahams BS, Herman EI, Winden KD, Gdalyahu A, et al: Absence of CNTNAP2 leads to epilepsy, neuronal migration abnormalities, and core autism-related deficits. Cell 147:235-246 (2011).

Peñagarikano O, Geschwind DH: What does CNTNAP2 reveal about autism spectrum disorder? Trends Mol Med 18:156-163 (2012).

$\checkmark$ Pinto D, Pagnamenta AT, Klei L, Anney R, Merico D, et al: Functional impact of global rare copy number variation in autism spectrum disorders. Nature 466:368-372 (2010).

- Poot M, Beyer V, Schwaab I, Damatova N, Van't Slot R, et al: Disruption of CNTNAP2 and additional structural genome changes in a boy with speech delay and autism spectrum disorder. Neurogenetics 11:81-89 (2010a).

- Poot M, Eleveld MJ, van't Slot R, Ploos van Amstel HK, Hochstenbach R: Recurrent copy number changes in mentally retarded children harbour genes involved in cellular localization and the glutamate receptor complex. Eur J Hum Genet 18:39-46 (2010b).

Poot M, van der Smagt JJ, Brilstra EH, Bourgeron T: Disentangling the myriad genomics of complex disorders, specifically focusing on autism, epilepsy, and schizophrenia. Cytogenet Genome Res 135:228-240 (2011).

-Salyakina D, Cukier HN, Lee JM, Sacharow S, Nations LD, et al: Copy number variants in extended autism spectrum disorder families reveal candidates potentially involved in autism risk. PLoS One 6:e26049 (2011).

-Sanders SJ, Ercan-Sencicek AG, Hus V, Luo R, Murtha MT, et al: Multiple recurrent de novo CNVs, including duplications of the $7 q 11.23$ Williams syndrome region, are strongly associated with autism. Neuron 70:863-885 (2011). Raubeson MJ, et al: De novo mutations revealed by whole-exome sequencing are strongly associated with autism. Nature 485: 237-241 (2012).

Schmeisser MJ, Ey E, Wegener S, Bockmann J, Stempel AV, et al: Autistic-like behaviours and hyperactivity in mice lacking ProSAP1/ Shank2. Nature 486:256-260 (2012).

Sebat J, Lakshmi B, Malhotra D, Troge J, LeseMartin C, et al: Strong association of de novo copy number mutations with autism. Science 316:445-449 (2007).

Shimoda Y, Koseki F, Itoh M, Toyoshima M, Watanabe K: A cis-complex of NB-2/contactin-5 with amyloid precursor-like protein 1 is localized on the presynaptic membrane. Neurosci Lett 510:148-153 (2012).

Skuse DH: Rethinking the nature of genetic vulnerability to autistic spectrum disorders. Trends Genet 23:387-395 (2007).

State MW, Levitt P: The conundrums of understanding genetic risks for autism spectrum disorders. Nat Neurosci 14:1499-1506 (2011).

Toro R, Konyukh M, Delorme R, Leblond C, Chaste $\mathrm{P}$, et al: Key role for gene dosage and synaptic homeostasis in autism spectrum disorders. Trends Genet 26:363-372 (2010).

Toyoshima M, Sakurai K, Shimazaki K, Takeda Y, Nakamoto $M$, et al: Preferential localization of neural cell recognition molecule NB-2 in developing glutamatergic neurons in the rat auditory brainstem. J Comp Neurol 513:349362 (2009).

Tropea D, Giacometti E, Wilson NR, Beard C, McCurry C, et al: Partial reversal of Rett Syndrome-like symptoms in MeCP2 mutant mice. Proc Natl Acad Sci USA 106:2029-2034 (2009).

Tsai PT, Hull C, Chu Y, Greene-Colozzi E, Sadowski AR, et al: Autistic-like behaviour and cerebellar dysfunction in Purkinje cell Tsc1 mutant mice. Nature 488:647-651 (2012).

van Daalen E, Kemner C, Verbeek NE, van der Zwaag B, Dijkhuizen T, et al: Social Responsiveness Scale-aided analysis of the clinical impact of copy number variations in autism. Neurogenetics 12:315-323 (2011).

van der Zwaag B, Staal WG, Hochstenbach R, Poot M, Spierenburg HA, et al: A co-segregating microduplication of chromosome 15q11.2 pinpoints two risk genes for autism spectrum disorder. Am J Med Genet B Neuropsychiatr Genet 153B:960-966 (2010).
Veenstra-VanderWeele J, Muller CL, Iwamoto H, Sauer JE, Owens WA, et al: Autism gene variant causes hyperserotonemia, serotonin receptor hypersensitivity, social impairment and repetitive behavior. Proc Natl Acad Sci USA 109:5469-5474 (2012).

Vernes SC, Newbury DF, Abrahams BS, Winchester L, Nicod J, et al: A functional genetic link between distinct developmental language disorders. N Engl J Med 359:2337-2345 (2008).

Vlamings PH, Jonkman LM, van Daalen E, van der Gaag RJ, Kemner C: Basic abnormalities in visual processing affect face processing at an early age in autism spectrum disorder. Biol Psychiatry 68:1107-1113 (2010).

Vorstman JA, Staal WG, van Daalen E, van Engeland $\mathrm{H}$, Hochstenbach PF, Franke L: Identification of novel autism candidate regions through analysis of reported cytogenetic abnormalities associated with autism. Mol Psychiatry 11:1, 18-28 (2006).

Wang K, Zhang H, Ma D, Bucan M, Glessner JT, et al: Common genetic variants on 5p14.1 associate with autism spectrum disorders. Nature 459:528-533 (2009).

-Won H, Lee HR, Gee HY, Mah W, Kim JI, et al: Autistic-like social behaviour in Shank2-mutant mice improved by restoring NMDA receptor function. Nature 486:261-265 (2012).

Xu JY, Xia QQ, Xia J: A review on the current neuroligin mouse models. Sheng Li Xue Bao 64: 550-562 (2012).

Yu TW, Chahrour MH, Coulter ME, Jiralerspong $\mathrm{S}$, Okamura-Ikeda $\mathrm{K}$, et al: Using wholeexome sequencing to identify inherited causes of autism. Neuron 77:259-273 (2013).

Zhang C, Atasoy D, Araç D, Yang X, Fucillo MV, et al: Neurexins physically and functionally interact with GABA(A) receptors. Neuron 66: 403-416 (2010).

Zhao X, Leotta A, Kustanovich V, Lajonchere C, Geschwind DH, et al: A unified genetic theory for sporadic and inherited autism. Proc Natl Acad Sci USA 104:12831-12836 (2007). 